\title{
Influence of fluid-structure interaction effects on the ductile fracture of blast-loaded steel plates
}

\author{
Vegard Aune ${ }^{1,2^{*}}$, Georgios Valsamos ${ }^{3}$, Folco Casadei $^{3 \dagger}$, Magnus Langseth ${ }^{1,2}$ and Tore Børvik ${ }^{1,2}$ \\ ${ }^{1}$ Structural Impact Laboratory (SIMLab), Department of Structural Engineering, NTNU - Norwegian \\ University of Science and Technology, Trondheim, Norway \\ ${ }^{2}$ Centre for Advanced Structural Analysis (CASA), NTNU, Trondheim, Norway \\ ${ }^{3}$ European Commission, Joint Research Centre (JRC), Ispra (VA), Italy
}

\begin{abstract}
This work presents ongoing research on the influence of fluidstructure interaction (FSI) effects on the ductile crack growth in perforated steel plates subjected to blast loading. The FSI effects were studied numerically by comparing the predictions from an uncoupled and a coupled FSI approach. Experimental results were used to evaluate the reliability of the numerical simulation. It was found that the numerical models were able to predict both crack initiation and crack growth in the plate, however, some distinct differences were also observed in the performance of the two approaches under consideration. The most important feature in predicting the observed fracture patterns was an accurate description of the blast loading during the FSI.
\end{abstract}

\section{Introduction}

One of the open research questions during severe blast loading events is the influence of fluid-structure interaction (FSI) effects on the dynamic fracture of lightweight and ductile structures [1,2]. Recent advancements [3,4] have managed to establish experimental and numerical methodologies allowing for detailed studies on the FSI during the dynamic response of blast-loaded steel plates. A combination of experiments and numerical simulations can therefore be used to obtain more insight into the underlying physics during extreme blast-structure interaction. For instance, it was shown in Ref. [4] that FSI produces a non-uniform spatial distribution of the time-dependent loading on the plate. The velocity induced in the plate tends to reduce the pressure during the first part of the FSI phase, while an increase in pressure magnitude due to the deformed shape of the plate was observed in the simulations during the second part of the FSI phase.

Although Ref. [5] provides some guidelines on the effect of one single perforation on the yield line locations in two-way supported plates, it is clearly stated that it is difficult to provide detailed guidance on the response of perforated plates. The effect of perforations on the dynamic response of blast-loaded plates is generally a function of location, size and shape. This motivates further studies on FSI effects during the dynamic response of even more complex structures. This work presents an experimental and numerical investigation on the influence of FSI on the ductile crack growth in a thin, perforated steel plate.

* Corresponding author: vegard.aune@ntnu.no

${ }^{\dagger}$ Active senior 


\section{Shock tube tests}

The blast test was carried out in the SIMLab Shock Tube Facility (SSTF) at NTNU, which has proven to generate controlled and repeatable blast-like loading conditions (see e.g. Ref. [3]). An illustration of the experimental setup, the clamping assembly and a picture of the test specimen are shown in Fig. 1. The experimental results presented in this study were already published in the work in Ref. [1], and the following presentation is therefore limited to a brief overview of the experimental setup.

The plate was manufactured from a sheet of Docol 600DL, had an exposed area of 300 $\mathrm{mm} \times 300 \mathrm{~mm}$, and the blast intensity was chosen such that the plate experienced large deformations (including ductile fracture). Geometrical defects were introduced in the plate prior to testing by wire erosion, initiating ductile fracture and crack propagation in the plate during testing. The defects were represented by square holes of $60 \mathrm{~mm}$ x $60 \mathrm{~mm}$ positioned symmetrically around the plate centre (Fig. 1b-c), resulting in a natural period of vibration $\left(T_{\mathrm{n}}\right)$ of $12.9 \mathrm{~ms}$. The blast intensity represents a peak reflected pressure of $1.1 \mathrm{MPa}\left(p_{\mathrm{r}, \max }\right)$ followed by an exponential decay over a period of $73.9 \mathrm{~ms}\left(t_{\mathrm{d}+}\right)$. This test is therefore in the dynamic loading regime $\left(0.064<T_{\mathrm{n}} / t_{\mathrm{d}+}<6.4\right)$, indicating that the peak pressure will dominate the dynamic response [6]. Two high-speed cameras (Phantom v1610) were positioned in a stereoscopic setup and used to capture the dynamic response of the plate, operating at a sampling rate of $24 \mathrm{kHz}$ (Fig. 1a). In addition to the blast test, uniaxial tensile tests were performed to determine the material behaviour (see Ref. [1]).

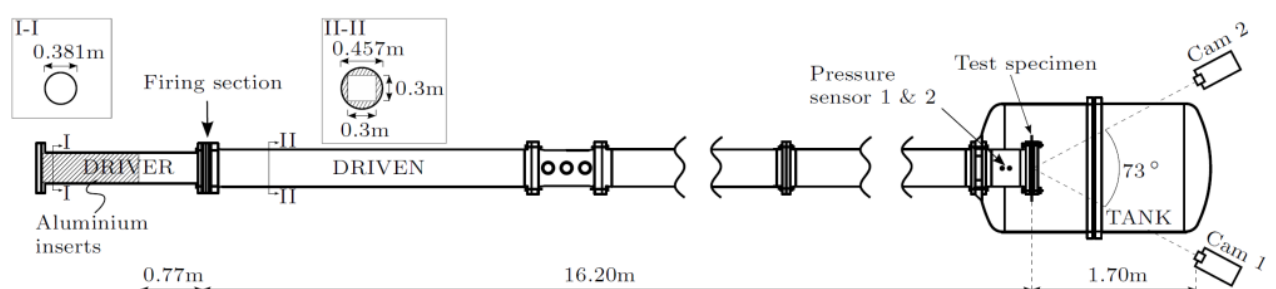

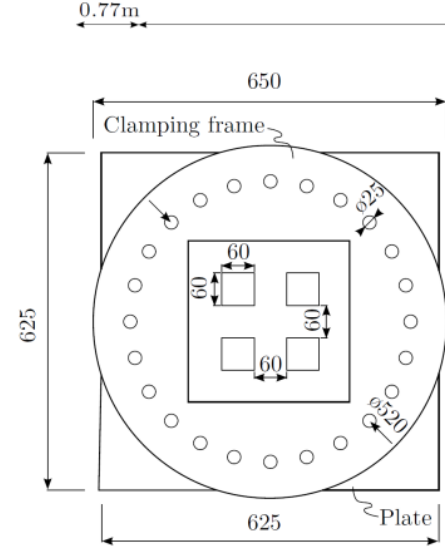

(b) (a)

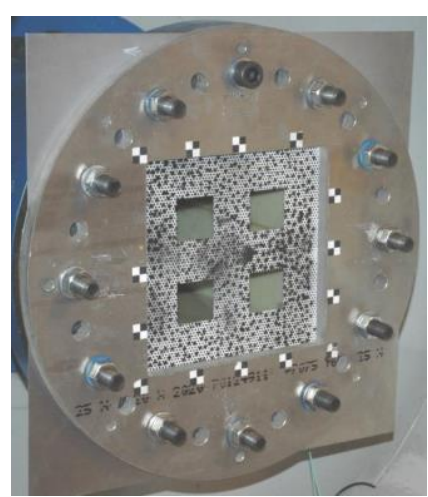

(c)

Fig. 1 Test setup: (a) Sketch of the setup, (b) dimensions (in mm) of the clamping assembly, and (c) image of the entire clamping and plate assembly. Both (b) and (c) are seen from the cameras. Reprint from Ref. [1].

\section{Computational methodology to study FSI effects}

The numerical simulations were performed by the explicit FE code EUROPLEXUS (EPX) [7]. FSI effects were studied using the numerical methodology presented in Ref. [4], while the high-speed images from the experiment were used as a backdrop to evaluate the predictive capabilities of the numerical models in capturing the actual physics of the problem. 
Fig. 2 depicts the assembly of the numerical model, where the symmetry planes in the experimental setup allowed to use only one quarter (1/4) of the complete assembly in Fig. 1. The fluid sub-domain was discretized with cell-centred finite volumes (CCFV), whereas the clamping assembly and the plate were discretized with solid elements (bricks CUB8 and wedges $P R O$ ) and $6 \mathrm{~mm}$ Reissner-Mindlin shell elements (quadrangles $Q 4 G S$ and triangles $T 3 G S$ ), respectively. Contact between the clamping assembly and the plate was modelled using a pinball contact model (see Ref. [1]). Coupling between the structural sub-domain and the fluid sub-domain was achieved by an FSI algorithm of the embedded (or immersed) type, known as FLSW in EPX (see Ref. [8]). FSI-driven adaptive mesh refinement (AMR) was used to refine the fluid mesh at the fluid-structure interface (see Ref. [4]). The material behaviour was modelled using a modified Johnson-Cook constitutive model (called VPJC in EPX) and ductile fracture was included using the Cockcroft-Latham (CL) fracture criterion [9]. Material parameters for the steel under consideration were taken from Ref. [1]. Damagebased AMR was activated in the plate using a threshold criterion on the CL fracture parameter, allowing for element erosion with limited loss of mass.

FSI effects were studied by comparing the numerical predictions of an uncoupled approach and a coupled (FSI) approach. The uncoupled approach contains only the clamping assembly and the plate in Fig. 2, where a pressure history is imposed on the blast-exposed area of the plate. This pressure history was obtained in a separate simulation containing only the fluid sub-domain where the plate is assumed to be a rigid wall with no perforations. Thus, the uncoupled simulations make the inherent assumption that the pressure is not influenced by the perforations and is unaltered by the structural motion, and vice versa. The uncoupled approach is therefore a conservative simplification of the real behaviour because it is expected to overestimate the actual pressure on the plate (see e.g. Ref. [4]).

Finally, a simulation following the coupled approach was carried out including the entire model in Fig. 2, i.e., both the fluid and structural sub-domain in the same simulation. This allowed for numerical investigations on FSI effects during the dynamic response of the plate, by comparisons to the numerical results obtained with the uncoupled approach.

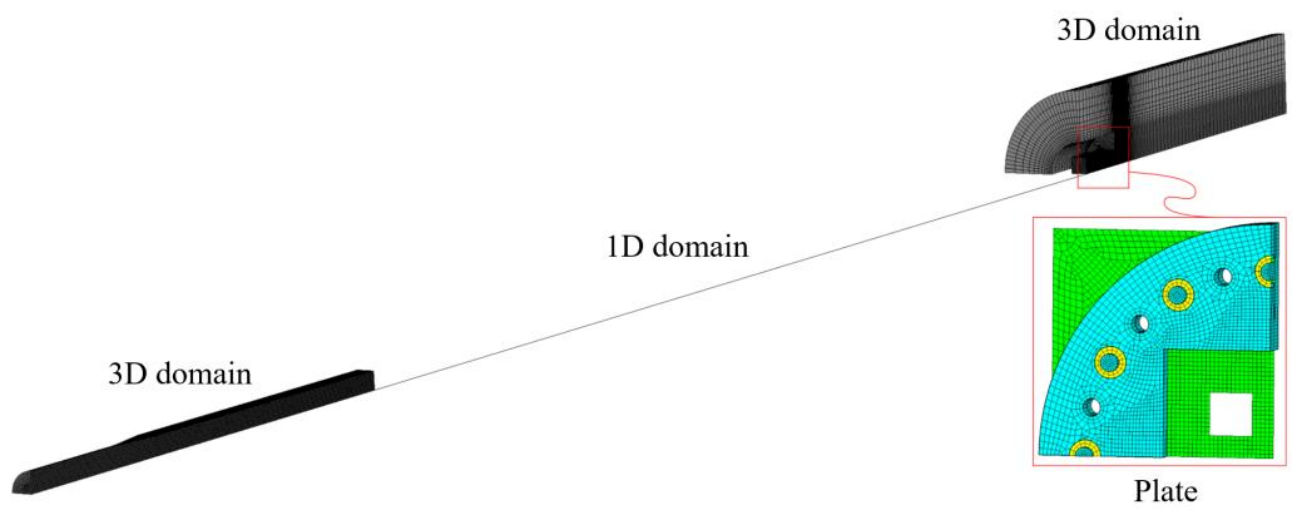

Fig. 2 Numerical model (1/4) of the fully coupled (FSI) approach. The clamping and plate assembly was also used (stand-alone) in the uncoupled approach. Inspired by Ref. [4].

\section{Results}

Fig. 3 shows a comparison of crack initiation and crack propagation during the experiment, the uncoupled simulation, and the coupled simulation. The comparisons are shown at characteristic times representing the overall trends in this study. Mirroring across the symmetry planes are used to illustrate the complete (4/4) model of the plate.

The effects of the perforations on the dynamic response of the plate are as expected and according to the guidance given in Ref. [5]. That is, yield lines form along the diagonals and 
define the crack paths. It is found that both the uncoupled and the coupled approach predict crack initiation and crack propagation in the plate, where the experimental observations are somewhere in-between the uncoupled and coupled simulations. In fact, it may seem that the coupled approach is somewhat closer to the experimental observations, at least in the initial part of the crack growth (see Figs. $3 \mathrm{a}$ and $3 \mathrm{~g}$ ). However, the cracks are arrested, and the coupled approach is therefore not able to predict the complete fracture of the plate (see Figs. $3 \mathrm{~b}$ and $3 \mathrm{~g}$ ). As expected, the uncoupled approach is conservative because it predicts faster crack propagation than the experimental observations (see Figs. 3a-c and 3d-f). It is important to keep in mind that the coupled simulation adds significant complexity compared to the uncoupled approach, and that the crack propagation is very sensitive to the magnitude of the loading. The results from this study show a noticeable influence of FSI on the crack growth in the plate. However, these results should be used with caution and detailed investigations on the performance of the coupled FSI model should be carried out to evaluate its reliability in predicting the actual loading during the experiments. Such investigations are beyond the scope of this study and are suggested as further work.

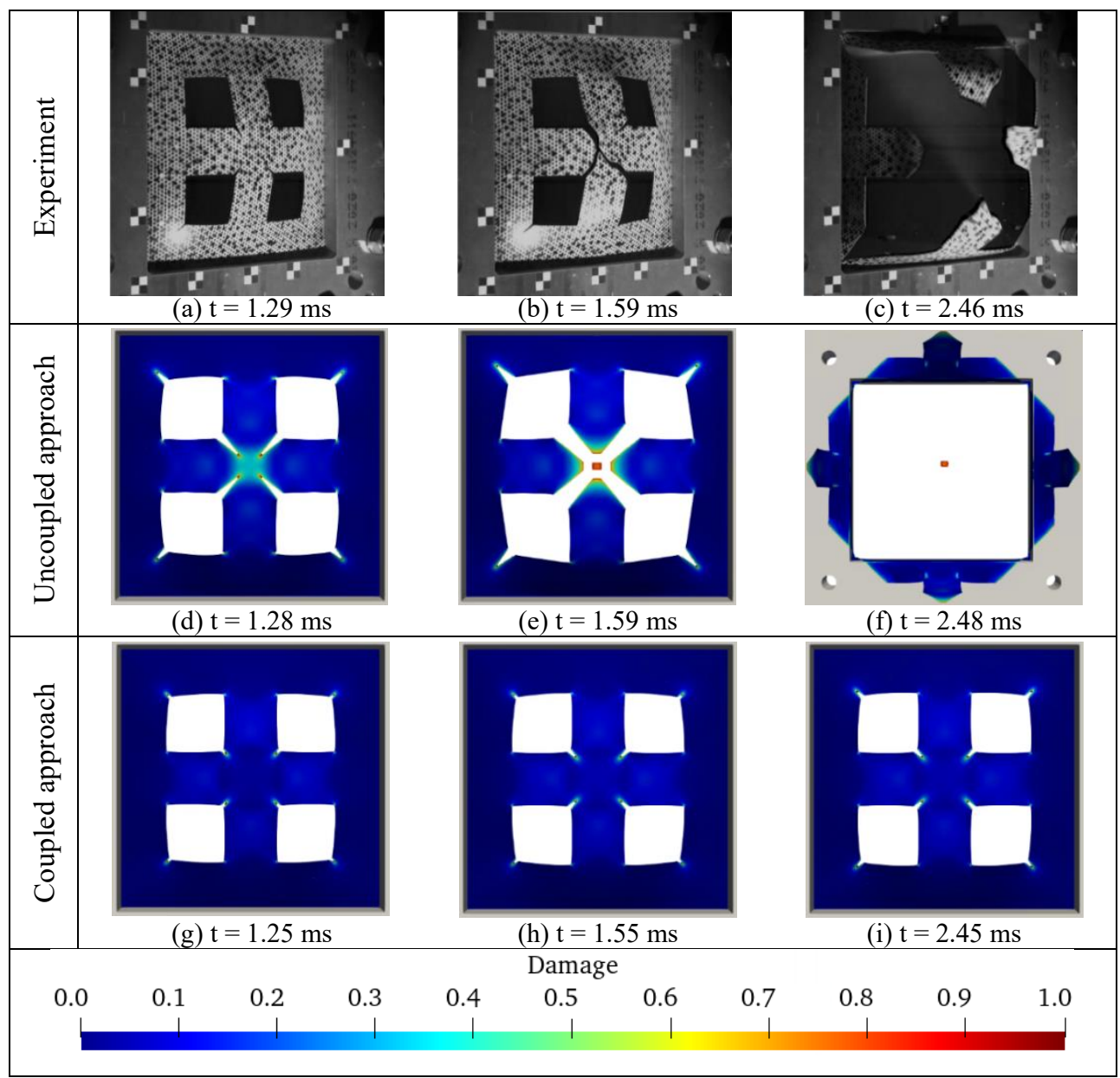

Fig. 3. Comparison between the experimental observations (top row) and the damage evolution in the uncoupled simulation (middle row) and coupled simulation (bottom row). Fringe colours represent the normalized damage in the plate. Time zero $(t=0)$ is taken as the arrival time of the shock wave at pressure sensor 1 located $24.5 \mathrm{~cm}$ upstream the test specimen (see Fig. 1 a). 
Fig. 4 shows a comparison of the pressure distribution on the plate for the uncoupled and the coupled (FSI) approach at the same characteristic times as in Fig. 3. Experimental pressure fields are not included because it is not possible to obtain these from the experimental data. To date, there is no measurement technique allowing to determine the full-field surface pressures during the dynamic response of flexible plates experiencing large deformations. The investigation of the surface pressures acting on the plate is therefore based only on the numerical results.

As expected, the pressure distribution becomes non-uniform during the dynamic response of the plate. It is also observed a focusing effect similar to that in Ref. [4] where, at some instants, the pressure at the plate center is higher in the coupled simulation (Fig. 4d) than in the uncoupled approach (Fig. 4a). Then, as the plate reaches its permanent deformation in the coupled approach, the pressure becomes lower than in the uncoupled approach (see Figs. 4b-c and 4e-f). It is important to emphasize that the uncoupled approach assumes that the pressure loading is unaltered by the fluid flow through the holes and by the deformation of the plate. Furthermore, the pressure in the uncoupled approach is always acting perpendicularly to the front surface of the plate. This implies that the applied pressure is overestimated since some fluid flow is vented into the tank (see Fig. 1). That is, in the uncoupled simulation the fracture mode is non-physical as the plate undergoes large displacements and large rotations (Figs. 3e-f and $4 \mathrm{~b}-\mathrm{c}$ ). If the plate is sufficiently deformed, one may end up in a situation where the load is acting in the opposite direction to the initial blast load (Fig. 3f). For these reasons it is expected that the coupled approach is closer to predicting the actual loading on the plate. Future studies will therefore focus on an improvement of the fluid sub-domain in predicting the actual loading, which will enable more detailed studies on the final shapes of the plates.

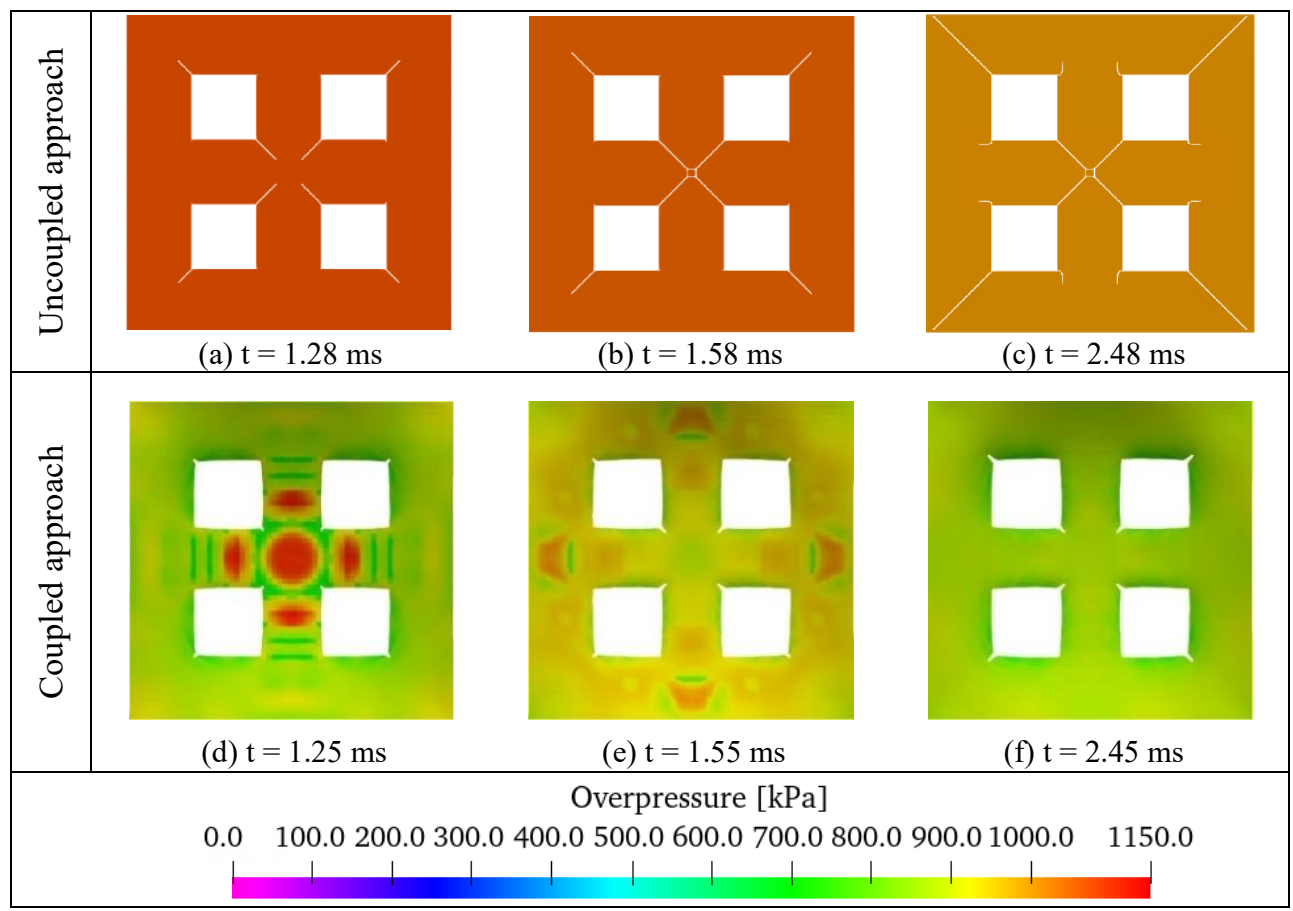

Fig. 4. Comparison of the pressure distribution on the plates for the uncoupled (top row) and the coupled (bottom row) approach at the same characteristic times as in Fig. 3. Fringe colours represent the contour map of the overpressure (in $\mathrm{kPa}$ ) acting on the plate. The pressure field for the uncoupled approach is obtained using the EFSI directive in EPX. The deformed geometry is mapped back to the initial configuration to better visualize the pressures during large deformations of the plate. 


\section{Concluding remarks}

This study investigates the influence of FSI effects on the dynamic response and fracture of blast-loaded steel plates. Such effects were studied by comparing the numerical predictions of the uncoupled and the coupled (FSI) approach. Special focus was placed on the influence of FSI on the ductile crack growth in the plates, where experimental data served as a backdrop to evaluate the accuracy of the numerical simulations. The main conclusions are summarized as follows.

- The clear conclusion from this study is that the ductile fracture and crack propagation are influenced by FSI effects during the dynamic response of the plate.

- FSI effects were observed on the ductile crack growth and as non-uniform spatial pressure distributions on the blast-exposed area of the plate.

- Although the crack initiation was similar in both the uncoupled and coupled simulations, the crack propagation showed some distinct differences. The coupled approach experienced crack arrest and was therefore not able to predict the complete fracture of the plate, whereas the uncoupled approach was conservative in predicting faster crack propagation than in the experimental observations.

- Comparison of numerical results and experimental observations indicates that the coupled (FSI) approach was very close to predict complete fracture, and that the crack growth was very sensitive to the actual loading on the plate. In the uncoupled simulations the applied loading becomes non-physical as the plate undergoes large displacements and large rotations, resulting in excessive fracture.

- It is emphasized that this work is ongoing research and that the presented results should be used with caution. More detailed studies on the reliability of the coupled (FSI) simulations are planned as further work. Still, the computational methodology used in this study has proven to be well suited to study the influence of FSI on the ductile crack growth in blast-loaded steel plates. This motivates similar studies on even more complex geometries.

\section{References}

1. V. Aune, G. Valsamos, F. Casadei, M. Langseth, T. Børvik. Int. J. Impact Eng. 108 (2017) 27-46.

2. H. Granum, V. Aune, T. Børvik, O. S. Hopperstad. Int. J. Impact Eng. 123 (2019) 103306.

3. V. Aune, E. Fagerholt, M. Langseth, T. Børvik. Int. J. Protective Struct. 7 (2016) 349366.

4. V. Aune, G. Valsamos, F. Casadei, M. Langseth, T. Børvik. Int. J. Mech. Sci. 195 (2021) 106263.

5. Department of Defense (DoD). Structures to Resist the Effects of Accidental Explosions (Report No. UFC 3-340-02, Washington, D.C.: Department of Defense, 2008).

6. W. E. Baker, P. A. Cox, P. S. Westine, J. J. Kulesz and R. A. Strehlow. Explosion Hazards and Evaluation (Elsevier, Amsterdam, 1983) p. 273-281.

7. EUROPLEXUS. http://europlexus.jrc.ec.europa.eu [accessed 08.04.2021].

8. F. Casadei, M. Larcher, N. Leconte. Strong and weak forms of a fully non-conforming FSI algorithm in fast transient dynamics for blast loading of structures, in Proceedings of the 3rd international conference on computational methods in structural dynamics and earthquake engineering, COMPDYN (2011) 1120-39.

9. M. G. Cockcroft, D. J. Latham. J. Inst. Met. 96 (1968) 33-39. 